\title{
Healthy Eating: Food Storage Guide ${ }^{1}$
}

\author{
Linda B. Bobroff and Jennifer Hillan²
}

If you don't shop for groceries often, you may want to buy food that will last until your next trip to the store. The following storage guidelines for perishable foods will help you decide how much food to buy when you visit the grocery. Keep in mind that safe storage times depend on the condition of the food when you purchase it and your refrigerator temperature and humidity. Check foods often for any signs of spoilage.

Tip: Store fruits and vegetables (except bananas, potatoes, and tomatoes) in the crisper drawer of your refrigerator, and keep your refrigerator at or below $40^{\circ} \mathrm{F}$. Bananas, potatoes, and tomatoes store best at room temperature. It is best to store fruits separate from vegetables.

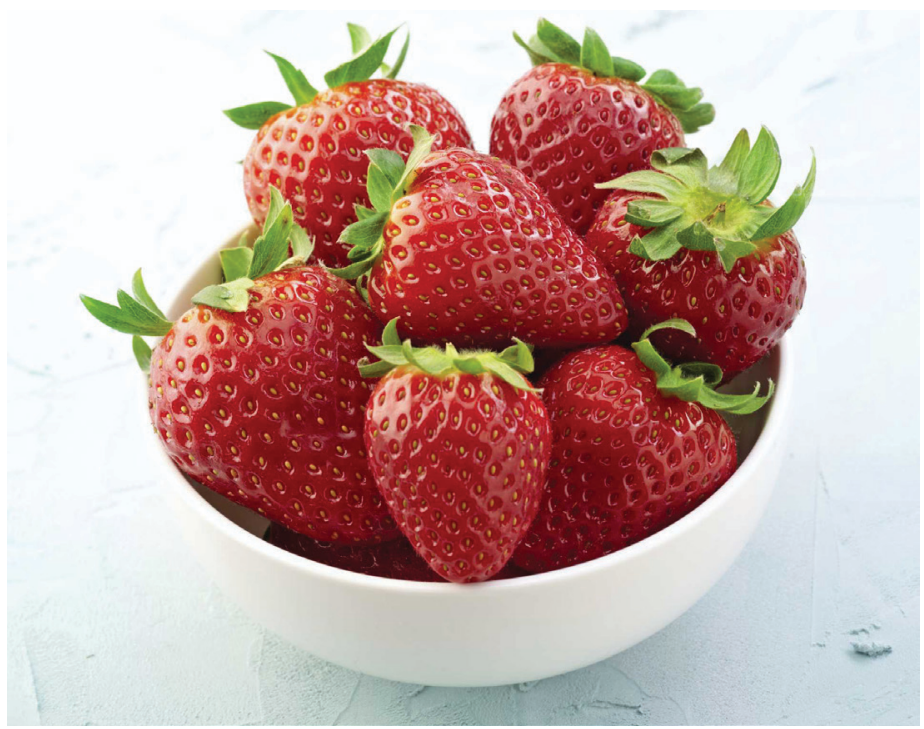

Figure 1. Credits: iStock
Table 1. Storage guidelines for selected perishable foods

\begin{tabular}{|c|c|}
\hline \multicolumn{2}{|l|}{ Fresh Fruit } \\
\hline $1-2$ days & Strawberries, raspberries, cherries \\
\hline $2-4$ days & $\begin{array}{l}\text { Apricots, bananas (room temperature), blueberries, } \\
\text { nectarines, peaches, pears, plums, melons } \\
\text { (cantaloupe, honeydew, watermelon, etc.) }\end{array}$ \\
\hline $1-2$ weeks & Grapes, citrus fruits, cranberries \\
\hline 3 weeks & Apples \\
\hline \multicolumn{2}{|c|}{ Fresh Vegetables } \\
\hline $1-2$ days & Corn on the cob, greens (spinach, kale, collards, etc.) \\
\hline 2-3 days & $\begin{array}{l}\text { Bok choy, mushrooms, okra, tomatoes (or until ripe; } \\
\text { store at room temperature) }\end{array}$ \\
\hline $3-5$ days & $\begin{array}{l}\text { Asparagus, bell pepper, broccoli, Brussels sprouts, } \\
\text { cauliflower, cucumber, eggplant, peas, green onion, } \\
\text { summer squash }\end{array}$ \\
\hline 1 week & Beans (snap or wax), celery, salad greens \\
\hline $1-2$ weeks & Cabbage, beets, iceberg lettuce, radishes \\
\hline 2-3 weeks & Carrots, parsnips, rutabagas, turnips \\
\hline 3-4 weeks & $\begin{array}{l}\text { Potatoes, sweet potatoes (store potatoes at room } \\
\text { temperature) }\end{array}$ \\
\hline \multicolumn{2}{|c|}{ Milk Products, Eggs, and Deli Meat } \\
\hline $3-5$ days & Sliced deli meat (once package is opened) \\
\hline 1 week & Milk, cottage cheese \\
\hline $1-2$ weeks & Yogurt \\
\hline 3-4 weeks & Hard cheese \\
\hline 3-5 weeks & Eggs, fresh, in shell \\
\hline
\end{tabular}

1. This document is FCS8695, one of a series of the Department of Family, Youth and Community Sciences, UF/IFAS Extension. First published: February 2005. Latest revision: October 2013. Please visit the EDIS website at http://edis.ifas.ufl.edu.

2. First edition adapted by Jennifer Hillan, MSH, RD, LD/N, former ENAFS nutrition educator/trainer, from the USDA publication, Shopping for Food and Making Meals in Minutes Using the Dietary Guidelines. Subsequent revisions by Linda B. Bobroff, PhD, RD, LD/N, professor, Department of Family, Youth and Community Sciences, UF/IFAS Extension, Gainesville, FL 32611.

The Institute of Food and Agricultural Sciences (IFAS) is an Equal Opportunity Institution authorized to provide research, educational information and other services only to individuals and institutions that function with non-discrimination with respect to race, creed, color, religion, age, disability, sex, sexual orientation, marital status, national origin, political opinions or affiliations. For more information on obtaining other UF/IFAS Extension publications, contact your county's UF/IFAS Extension office. 


\section{References}

Food Marketing Institute. (2013). Food Keeper. Retrieved from http://www.fmi.org/consumer/foodkeeper/

Food and Drug Administration. (2013). Refrigerator \& Freezer Storage Chart. Retrieved from http://www.fda. gov/downloads/Food/FoodborneIllnessContaminants/ ucm109315.pdf
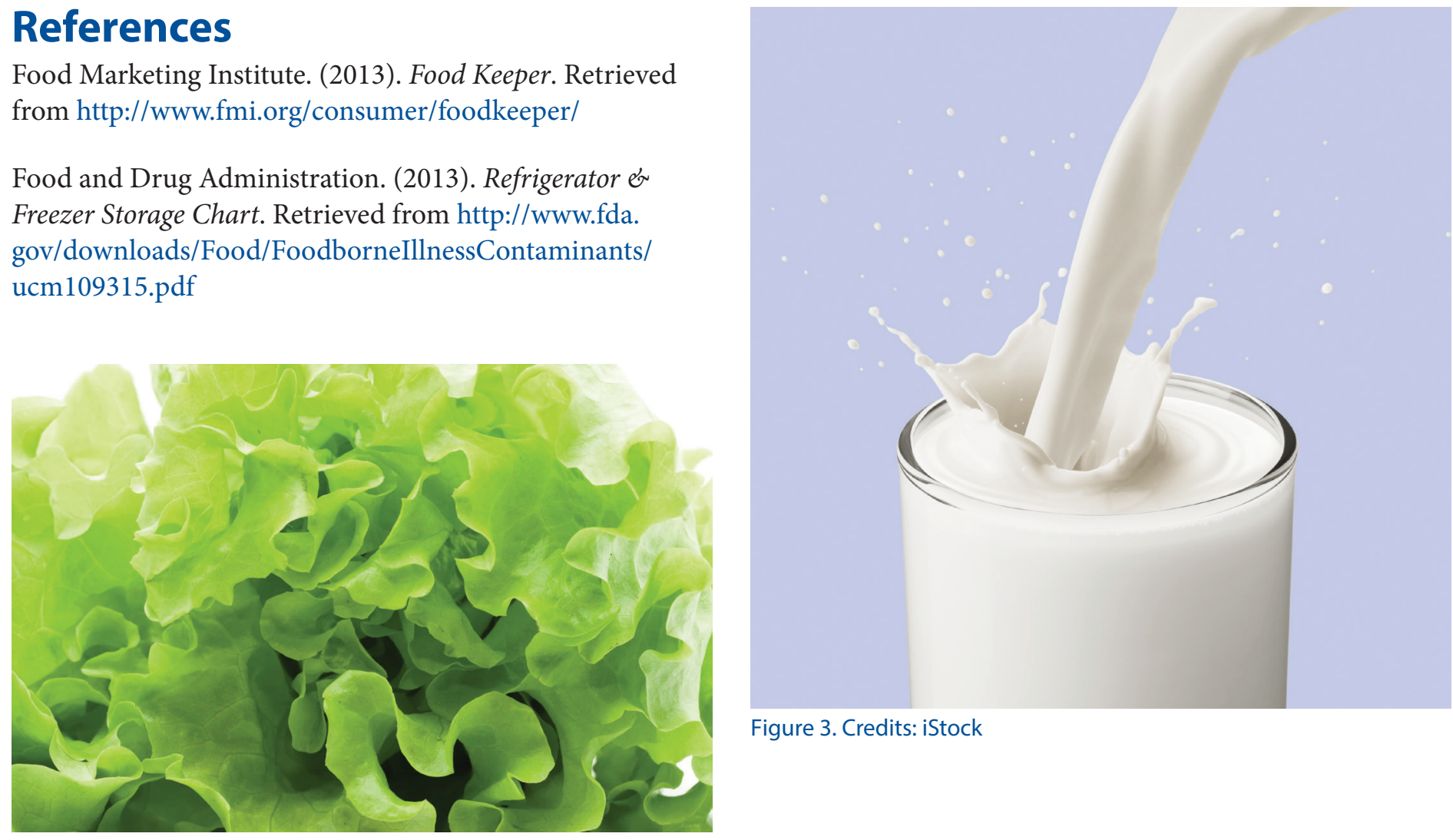

Figure 3. Credits: iStock

Figure 2. Credits: iStock 